\title{
NONCONJUGATE POINTED GENERALIZED SOLENOIDS WITH SHIFT EQUIVALENT $\pi_{1}$-ACTIONS
}

\author{
RICHARD SWANSON
}

(Communicated by Bryna Kra)

\begin{abstract}
Going back to the pioneering work of R. F. Williams, it has been widely believed that if elementary presentations $\left(K_{i}, f_{i}\right), i=1,2$, satisfy the Williams axioms and fix the branch points, then there is a pointed conjugacy

$$
\bar{r}:\left(\lim _{\longleftarrow}\left(K_{1}, f_{1}\right), \bar{y}_{1}\right) \rightarrow\left(\lim _{\longleftarrow}\left(K_{2}, f_{2}\right), \bar{y}_{2}\right)
$$

between the natural shift maps $\bar{f}_{1}$ and $\bar{f}_{2}$ if and only if the fundamental group homomorphisms $\pi_{1}\left(f_{1}, y_{1}\right)$ and $\pi_{1}\left(f_{2}, y_{2}\right)$ are shift equivalent. The "only if" direction is true. We prove that the "if" direction goal of this belief is false by exhibiting a family of counterexamples not previously analyzed. Of course, the associated hyperbolic attractors constructed by R. F. Williams cannot be conjugate in these exceptional cases.
\end{abstract}

A presentation is a mapping pair $(K, f)$ such that $f: K \rightarrow K$ is a continuous endomorphism of a graph $K$, with inverse limit space $\lim _{\longleftarrow}(f, K)$ whose shift map $\bar{f}$ is conjugate to the given homeomorphism on $\mathcal{S}$, a generalized solenoid. Williams showed (in [5, Theorem 3.3]) that two shift maps $\bar{f}_{1}$ and $\bar{f}_{2}$, on presentations $\left(K_{1}, f_{1}\right)$ and $\left(K_{2}, f_{2}\right)$, are topologically conjugate if and only if the maps $f_{1}$ and $f_{2}$ are shift equivalent. He was able to show further that shift equivalence is equivalent to "strong shift equivalence" in the category of maps on branched 1-manifolds satisfying the Williams axioms (see [2] or Axioms 2.1 in [5]). This reduces checking shift equivalence to seeking a sequence of "elementary" (or lag 1) shift equivalences.

Williams attempted to link the shift equivalence of pointed presentations (corresponding to pointed conjugacy classes of shifts $\bar{f}:\left(\lim _{(K, f)}(K) \rightarrow\left(\lim _{\leftarrow}(K, f), \bar{x}\right)\right.$, $\bar{x}=(x, x, \ldots)$ to the shift equivalence of $\pi_{1}$ representations. Williams defines the shift class $S(\bar{f}, x)$ of $\bar{f}$ to be the shift equivalence class of $\pi_{1}(f, x): \pi_{1}(K, x) \rightarrow$ $\pi_{1}(K, x)$. A presentation $(K, f)$ is elementary if $K$ is a wedge of circles and $f$ fixes the branch point $y$ of $K$.

For some time, it has been generally believed (e.g. [5, (7.3)], 44, (e.g. Remark 4.16)] and [2, ("Theorem")]) that if elementary presentations $\left(K_{i}, f_{i}\right), i=1,2$, satisfy the Williams axioms and $f_{i}\left(y_{i}\right)=y_{i}, i=1,2$, then there is a pointed conjugacy

$$
\bar{r}:\left(\lim _{\longleftarrow}\left(K_{1}, f_{1}\right), \bar{y}_{1}\right) \rightarrow\left(\lim _{\longleftarrow}\left(K_{2}, f_{2}\right), \bar{y}_{2}\right)
$$

of $\bar{f}_{1}$ with $\bar{f}_{2}$ if and only if the fundamental group homomorphisms $\pi_{1}\left(f_{1}, y_{1}\right)$ and $\pi_{1}\left(f_{2}, y_{2}\right)$ are shift equivalent. One goal of this paper is to show that this belief is

Received by the editors November 20, 2010 and, in revised form, April 11, 2001 and April 17, 2011.

2010 Mathematics Subject Classification. Primary 37B10, 37B45, 37B50; Secondary 37B99, $05 \mathrm{C} 20$. 
false. The existence of such an example, mentioned without details, was asserted in [3]. The author would like to thank M. Barge for helpful conversations.

Remark. A crucial element here is that the pointed conjugacy must preserve the fixed points corresponding to branch points of the wedge of circles.

Theorem 1. There exists a pair of elementary presentations, preserving branch points and satisfying the Williams axioms, having nonconjugate pointed shift maps yet whose fundamental group endomorphisms are strong shift equivalent.

Proof. Consider the presentations given by the wrapping rules

$$
\psi:\left\{\begin{array}{l}
a \mapsto a b b b b c \\
b \mapsto a b c \\
c \mapsto a c
\end{array} \quad \varphi:\left\{\begin{array}{l}
a \mapsto a a b b b b \\
b \mapsto a b
\end{array}\right.\right.
$$

that generate pointed graph maps $f=f_{\psi}:\left(K_{3}, p\right) \rightarrow\left(K_{3}, p\right)$ and $g=g_{\varphi}$ : $\left(K_{2}, q\right) \rightarrow\left(K_{2}, q\right)$, where $K_{3}$ is a wedge of 3 circles, $K_{2}$ of 2 circles and $p, q$ are the branch points. In the proof of Proposition 4.7 of [2, we prove that there is no shift equivalence $(r, s)$ between these maps such that $r(p)=q$. That is, there is no conjugacy of $\bar{f}$ with $\bar{g}$ that takes $\bar{p}$ to $\bar{q}$.

However, there is an explicit shift equivalence of lag 3 between the group endomorphisms $\pi_{1}(f, p)$ and $\pi_{1}(g, q)$ :

$$
\rho:\left\{\begin{array}{l}
a \mapsto b^{-1} a b b b b a b a b a b a b a b \\
b \mapsto b^{-1} a b b b b a b a b \\
c \mapsto b^{-1} a b b b b a b
\end{array} \quad \sigma:\left\{\begin{array}{l}
a \mapsto c^{-1} b c a c a b b b c \\
b \mapsto c^{-1} b c a c
\end{array}\right.\right.
$$

That is, $\varphi^{3}=\rho \circ \sigma$ and $\psi^{3}=\sigma \circ \rho$.

This is nontrivial to check directly, and, indeed, the author derived the connecting maps by computing products $\rho=\rho_{3} \rho_{2} \rho_{1}$ and $\sigma=\sigma_{1} \sigma_{2} \sigma_{3}$ (see below). Thus, this is a strong shift equivalence, because it passes through a series of 3 elementary (lag 1) shift equivalences that are considerably easier to establish.

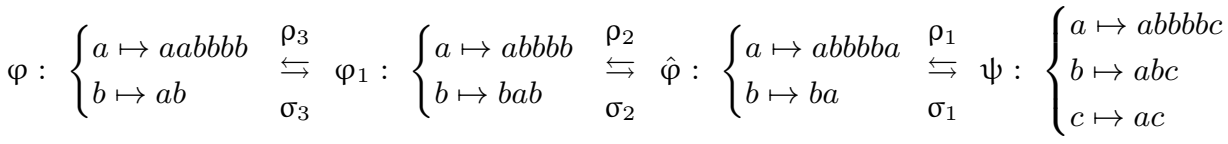

$$
\begin{aligned}
& \rho_{3}:\left\{\begin{array}{l}
a \mapsto b^{-1} a b b b b \\
b \mapsto a b
\end{array} \quad \rho_{2}:\left\{\begin{array}{l}
a \mapsto a b \\
b \mapsto b
\end{array} \quad \rho_{1}:\left\{\begin{array}{l}
a \mapsto a b b b b \\
b \mapsto a b \\
c \mapsto a
\end{array}\right.\right.\right. \\
& \sigma_{3}:\left\{\begin{array}{l}
a \mapsto b a \\
b \mapsto b
\end{array} \quad \sigma_{2}:\left\{\begin{array}{l}
a \mapsto a b b b \\
b \mapsto b a
\end{array} \quad \sigma_{1}:\left\{\begin{array}{l}
a \mapsto a c \\
b \mapsto c^{-1} b c
\end{array}\right.\right.\right.
\end{aligned}
$$

In this second part of the proof we must show that the shift maps $\bar{f}$ and $\bar{g}$ are not conjugate by a conjugacy that preserves the branch points. The easiest way to do this is to use the theorem of Williams that asserts it suffices to show the graph maps $f$ and $g$ are not shift equivalent. As in [2], we can rule out such a graph shift equivalence by showing that there is no symbolic substitution $\sigma$ that extends to carry the (unique) fixed word $w_{\varphi}$ of the substitution $\varphi$ over to the fixed word $w_{\psi}$ 
of $\psi$. These bi-infinite words are unique, since the substitutions are proper (e.g. [1]):

$$
\mathrm{w}_{\varphi}=\cdots a b \cdot a^{2} b^{4} \cdots \quad \text { and } \quad \mathrm{w}_{\psi}=\cdots a c \cdot a b b b b c \ldots .
$$

The substitution $\sigma$ can be assumed to be orientation-preserving with the right fixed word

$$
\sigma: \mathrm{w}_{\varphi}^{+}=a^{2} b^{4} \cdots \mapsto \mathrm{w}_{\psi}^{+}=a b b b b c \cdots
$$

Claim. $\mathrm{w}_{\psi}^{+}$does not have a repeated prefix $u u=\sigma(a) \sigma(a)$.

Proof of Claim. Let $u$ be the prefix of least length that is repeated in $\mathrm{w}_{\psi}^{+}$. The word $u$ begins with $a$. The only pairs that can occur in the fixed word are $a b, a c, b c$, and $c a$. Thus the last letter of $u$ must be $c$. This means that $u$ is the image of a prefix $v$ of $\mathrm{w}_{\psi}^{+}$. Now $\psi$ stretches words by the factor $\lambda=3.56 \ldots$ So $v$ is a repeated prefix shorter than $u$, contradicting our assumption.

It is worth examining the fundamental group shift equivalence constructed above. Call it $\widetilde{\sigma}$. Then

$$
\widetilde{\sigma}\left(a^{2}\right)=\left(c^{-1}(b c a c a b b b) c\right)^{2}=c^{-1}(b c a c a b b b)^{2} c .
$$

The result does have a repeated prefix taking the form of a conjugate in the fundamental group. Iterates by $\psi$ of $c^{-1} x$ for any word $x$ starting with $a$ or $b$ converge to the same infinite fixed word. If $x$ is empty, we get the unique orientation-reversed fixed word: $\lim \psi^{n}\left(c^{-1}\right)$, derived from the generators $\left\{a^{-1}, b^{-1}, c^{-1}\right\}$.

\section{WAys to move the Base POINT}

A key to understanding examples as in the preceding section is the concept of moving the base point. Roughly, this means that in a graph presentation of a solenoid, we do two things. Let $p$ denote a branch point and $x$ a fixed point not equal to the branch point. The solenoid would have fixed points $\bar{x}=(x, x, \ldots)$ and $\bar{p}=(p, p, \ldots)$. We consider loops in the graph that exit from and return to $x$. We iterate the graph map on these loops to find an invariant set of labeled generating loops. Next we replace the graph by a bouquet of circles with each circle corresponding exactly to one of the labeled return loops to the fixed point. There is an induced endomorphism on this latter graph. That is, the fixed point is now, symbolically, the branch point and the original branch point becomes a fixed point.

Given an elementary presentation of a Williams solenoid as the inverse limit of a graph endomorphism, there seem to be two different ways to move the base point, or branch point, if you like, to obtain a new elementary presentation.

(a) One can move the point topologically via an orientation-preserving return rewriting, which does not change the homeomorphism type of the solenoid (e.g. 1], 2]).

(b) One can move the base point algebraically via a path conjugacy followed by homotopy cancellations. This may change the topology of the induced solenoid, because of the cancellations.

We want to describe these procedures in a little more detail. 
1.1. Moving the branch point topologically. Split the loop $a_{i}$ as the composite $a_{i}=b_{1} \cdot b_{2}$ of an arc $b_{1}$ from $p$ to $x$ and $b_{2}$ from $x$ to $p$, each oriented the same as $a_{i}$. Now we replace the alphabet $\mathcal{A}$ with the (finite) return word alphabet $\mathcal{B}_{x}=\left\{B_{k}\right\}$ derived as follows: The image $f_{\varphi}\left(b_{2} b_{1}\right)$ must factor uniquely into words of the form $A_{k}=b_{2} w_{k} b_{1}$ provided we break at every occurrence of the subword $b_{1} b_{2}$. In a similar fashion, the image of each $B_{k}$ factors into words $A_{k j}=b_{2} w_{k j} b_{1}$. Keep iterating on any new return words that appear. This process must stop (e.g. [1) with a unique finite collection of words $B_{k}=b_{2} u_{k} b_{1}$ that form the alphabet for a unique substitution. It is not our main object here, but we observe that the tiling space based on this induced substitution in the alphabet $\mathcal{B}_{x}$ is homeomorphic to the original tiling space ([1]). A more general class of so-called rewritings of a substitution, called "starting and stopping rules", is analyzed in [1. The solenoids based on $\mathcal{A}$ and $\mathcal{B}_{x}$ are homeomorphic to each other and to the tiling spaces if the original substitution satisfies the Williams axioms (stronger than needed [2]). The tiling space homeomorphism is actually a flow equivalence of the translation flow on the tiling space.

\subsection{Moving the branch point algebraically.}

Theorem 2. Suppose $f$ and $g$ are elementary presentations with branch points $p$ and $q$ that follow the pattern of $\varphi=\pi_{1}(f, p)$ and $\psi=\pi_{1}(g, q)$. Suppose also that $g$ is obtained by moving the base point $p$ of $\varphi$ topologically to a fixed point $x$ and $\hat{f}$ is obtained by moving the base point algebraically to $x$. Then $\hat{\varphi}=\pi_{1}(\hat{f}, \hat{p})$ and $\psi=\pi_{1}(g, p)$ ( $p$ is the branch point of the wedge of circles that $g$ acts on) are shift equivalent free group endomorphisms.

Proof. We prove this for two symbols, as the general proof is an inductive extension. Given a primitive substitution morphism $\varphi$ acting on the letters $a$ and $b$, cut the loop $a=a_{1} a_{2}$ at a fixed point of the map $f$ that follows the pattern of $\varphi$ on a wedge of two circles. Algebraically rewrite with loops $\hat{a}=a_{1}^{-1} a a_{1}$ and $\hat{b}=a_{1}^{-1} b a_{1}$ (allow homotopy canceling of paths). This produces a substitution morphism in the letters $\hat{a}$ and $\hat{b}$. It is easy to check that the substitution $\hat{\varphi}$ can always be obtained by "cycling" $\varphi(a)$ and $\varphi(b)$ (move the first letter to the last position in each of those words, then put a hat on them).

Now rewrite, cutting $a=a_{1} a_{2}$ at the fixed point. These loops have the form $a_{2} b^{k} a_{1}$, where $b^{0}$ is ignored. Let $\psi$ denote the return substitution (or [start, stop] $=$ $\left.\left[a_{2}, a_{1}\right]\right)$ to $a_{2}$ in the fixed word starting with $a_{2}$.

We can associate $B_{0}=A=a_{2} a_{1}$ with the path-conjugate word $\hat{a}$ and, in general, the return word to the cut given by $B_{k}=a_{2} b^{k} a_{1}$ corresponds to $\hat{a} \hat{b}^{k}$. For ease in reading, unhat the hatted letters. This is one direction $\rho$ of an elementary shift equivalence: $\rho\left(B_{k}\right)=a b^{k}$. Of course, we may not need very many of the symbols $B_{k}$ (see example that follows). The morphism $\rho$ never reverses directions. We need to solve for the morphism $\sigma$ having the following properties:

$$
\begin{aligned}
& \sigma \rho\left(B_{0}\right)=\sigma(a):=\psi\left(B_{0}\right), \\
& \sigma\left(\rho\left(B_{1}\right)=\sigma(a b)=\psi\left(B_{0}\right) \sigma(b)=\psi\left(B_{1}\right) \Longrightarrow \sigma(b)=\psi\left(B_{0}^{-1} B_{1}\right),\right. \\
& \hat{\varphi} \rho B_{k}=\hat{\varphi}\left(a b^{k}\right)=\hat{\varphi} a(\hat{\varphi}(b))^{k}=\rho \psi\left(B_{k}\right) .
\end{aligned}
$$

It is the definition of $\sigma$ that can yield a morphism with conjugates and results in a fundamental group shift equivalence that is not a semigroup shift equivalence. 
Let us illustrate Proposition 2 with the semigroup endomorphism $\varphi$ defined in Theorem 1. We notice that the induced graph endomorphism $f=f_{\varphi}$ has a fixed point in loop $a$. Split $a$ at the fixed point $x$ such that $a=a_{1} a_{2}$. This induces wrapping rules $f\left(a_{1}\right)=a_{1} a_{2} a_{1}$ and $f\left(a_{2}\right)=a_{2} b b b b$. Maintaining orientation, we iterate on loops starting at $x$ and factor them:

$$
\begin{aligned}
f\left(a_{2} a_{1}\right) & =a_{2} b^{4} a_{1} a_{2} a_{1}=\left(a_{2} b^{4} a_{1}\right)\left(a_{2} a_{1}\right), \\
f\left(a_{2} b^{4} a_{1}\right) & =a_{2} b^{4} a_{1} a_{2} b a_{1} a_{2} b a_{1} a_{2} b a_{1} a_{2} b a_{1} a_{2} a_{1} \\
& =\left(a_{2} b^{4} a_{1}\right)\left(a_{2} b a_{1}\right)\left(a_{2} b a_{1}\right)\left(a_{2} b a_{1}\left(a_{2} b a_{1}\right)\left(a_{2} a_{1}\right),\right. \\
f\left(a_{2} b a_{1}\right) & =a_{2} b^{4} a_{1} a_{2} b a_{1} a_{2} a_{1}=\left(a_{2} b^{4}\left(a_{1} a_{2} b a_{1}\right)\left(a_{2} a_{1}\right) .\right.
\end{aligned}
$$

We rewrite the wrapping rules based on the new loops $a=a_{2} b b b b a_{1}, b=a_{2} b a_{1}$ and $c=a_{2} a_{1}$ to obtain

$$
\widetilde{\psi}:\left\{\begin{array}{l}
a \mapsto a b b b b c \\
b \mapsto a b c \\
c \mapsto a c .
\end{array}\right.
$$

Now this $\widetilde{\psi}$ has a different ordering of symbols from the substitution $\psi$ obtained in the proof of the theorem above, which is why a tilde is necessary. We will take care of this discrepancy below.

This is a topological rewriting that moves the base point and preserves orientation.

An algebraic way to rewrite the morphism $\varphi$ is to move the base point by a conjugacy rather than a return map. So with $a_{1}$ and $a_{2}$ as above, we let $\hat{a}=a_{1}^{-1} a a_{1}$ and $\hat{b}=a_{1}^{-1} b a_{1}$. Iterate $f$ on these algebraic loops and allow homotopy cancellation:

$$
\begin{aligned}
& \pi_{1}(f)(\hat{a})=\pi_{1}(f)\left(a_{2} a_{1}\right)=a_{2} b^{4} a_{1} a_{2} a_{1} \sim a_{1}^{-1} a b^{4} a a_{1} \sim \hat{a} \hat{b} \hat{b} \hat{b} \hat{b} \hat{a} \\
& \pi_{1}(f)(\hat{b}) \sim a_{1}^{-1} b a a_{1} \sim \hat{b} \hat{a} .
\end{aligned}
$$

Now replace $\hat{a}$ and $\hat{b}$ by $a$ and $b$. This is just the substitution $\hat{\varphi}$ given above. The previous argument shows that there is free group shift equivalence between the $\pi_{1}$ presentations of $\hat{\varphi}$ and $\psi$. This is given by the pair $\rho_{1}$ and $\sigma_{1}$ in the foregoing proof.

Notice that the morphism $\sigma_{1}$ is not a semigroup morphism. It requires a temporary reversal of order as $b$ maps to its conjugate by $c$. That is a general fact, which underlies the possibility of examples such as $\varphi$ and $\psi$. Following the algorithm of the proof above,

$$
\begin{aligned}
& \rho\left(B_{k}\right)=a b^{k} \quad \text { and, hence, } \\
& \sigma \rho\left(B_{0}\right)=\sigma(a)=\psi\left(B_{0}\right)=B_{4} B_{0}, \\
& \sigma \rho\left(B_{1}\right)=\sigma(a b)=\sigma(a) \sigma(b)=B_{4} B_{0} \sigma(b)=\psi\left(B_{1}\right)=B_{4} B_{1} B_{0} .
\end{aligned}
$$

So $\sigma(b)=B_{0}^{-1} B_{1} B_{0}$ and letting $a=B_{4}, b=B_{1}, c=B_{0}$ yields the connecting morphisms $\sigma_{1}$ and $\rho_{1}$ in the proof of Theorem 1 . 


\section{EXAMPLES AND A QUESTION}

It is not hard to see that moving the base point two different ways yields a family of examples similar to Theorem 1. These would have the form

$$
\psi:\left\{\begin{array}{l}
a \mapsto a b^{n} c \\
b \mapsto a b c \\
c \mapsto a c
\end{array} \quad \varphi:\left\{\begin{array}{l}
a \mapsto a a b^{n} \\
b \mapsto a b
\end{array} \quad \widehat{\varphi}:\left\{\begin{array}{l}
a \mapsto a b^{n} a \\
b \mapsto b a
\end{array}\right.\right.\right.
$$

We also have the example

$$
\psi:\left\{\begin{array}{l}
a \mapsto a b c \\
b \mapsto a b b c \\
c \mapsto a c
\end{array} \quad \varphi:\left\{\begin{array}{l}
a \mapsto a a b \\
b \mapsto a b b
\end{array} \quad \widehat{\varphi}:\left\{\begin{array}{l}
a \mapsto a b a \\
b \mapsto b b a
\end{array}\right.\right.\right.
$$

where the wrapping rules, $\psi$ and $\widehat{\varphi}$, are obtained from $\varphi$ by, respectively, moving the base point topologically and algebraically. This time the substitutions $\psi$ and $\varphi$ are not even shift equivalent over the fundamental groups.

Question. In Theorem 1, there is a conjugacy between the shift maps $\bar{f}$ and $\bar{g}$. It just does not preserve the branch point. This leaves the following question open: Is it possible, in the context of elementary presentations satisfying the Williams axioms, to have $\pi_{1}\left(f_{1}, y_{1}\right)$ shift equivalent to $\pi_{1}\left(f_{2}, y_{2}\right)$, while $\bar{f}_{1}$ is not conjugate to $\bar{f}_{2}$ ?

\section{REFERENCES}

[1] M. Barge and B. Diamond, A complete invariant for the topology of one-dimensional substitution tiling spaces, Ergod. Thy. and Dynam. Sys, 21, no. 5 (2001) 1333-1358. MR.1855835 (2002k:37026)

[2] M. Barge and R. Swanson, New techniques for classifying Williams solenoids, Tokyo J. of Math., 30, no. 1 (2007), 139-157. MR2328068 (2008f:37024)

[3] Erratum, Tokyo J. of Math., 34, no. 1 (2011), 287-288. MR.2866647

[4] I. Yi, Canonical symbolic dynamics for one-dimensional generalized solenoids, Trans. Amer. Math. Soc., 353 (2001), 3741-3767. MR.1837257 (2002h:37021)

[5] R. F. Williams, Classification of 1-dimensional attractors. Proc. Symp. Pure Math., 14, Amer. Math. Soc., 1970, pp. 341-361. MR0266227(42:1134)

Department of Mathematical Sciences, Montana State University, Bozeman, MonTANA 59717-2400 